\title{
PISCINE MYCOBACTERIOSIS IN GOLD FISH (CARASSIUS AURATUS) AND KOI CARP (CYPRINUS CARPIO): TWO CASE REPORTS
}

\author{
S.S.S. de S. Jagoda ${ }^{1}$, D.M.S.G. Dissanayake ${ }^{1}$, R.A.D.S. Ranathunga ${ }^{1}$, \\ D.R.A. Dissanayake' ${ }^{2}$ A. Arulkanthan ${ }^{1}$
${ }^{\prime}$ Center for Aquatic Animal Disease Diagnosis and Research, Department of Veterinary Pathobiology, Faculty of Veterinary Medicine and Animal Science, University of Peradeniya, Peradeniya 20400
${ }^{2}$ Veterinary Teaching Hospital, Department of Veterinary Clinical Sciences, Faculty of Veterinary Medicine and Animal Science, University of Peradeniya, Peradeniya20400

\begin{abstract}
SUMMARY: Piscine mycobacteriosis is a systemic, chronic, progressive disease in fish caused by the nontuberculous mycobacteria (NTM). It has significant economic and public health impacts on commercial culture of ornamental fish. Clinical manifestation and the pathology of the infection in ornamental fish may vary depending on the species of fish affected and the species of the pathogenic NTM involved. This clinical communication describes mycobacteriosis in two different ornamental species; gold fish (Carassius auratus) and koi carp (Cyprinus carpio).
\end{abstract}

\section{BACKGROUND}

Piscine Mycobacteriosis is a chronic progressive disease affecting many species of wild and cultured fish inhabiting both fresh water and marine environments (Jacobs, 2009; Gauthier and Rhodes, 2009). It is considered as the most prevalent chronic disease in tropical and subtropical fresh water aquarium/ornamental fish (Antonio et al., 2000; Gauthier and Rhodes, 2009) and has been reported in native and imported ornamental fish stocks in many countries including Thailand, Italy, Czech Republic, Slovenia, Poland, Iran, India and Sweden. An increasingly diverse array of Mycobacterium spp., has been implicated in piscine mycobacteriosis (Gauthier and Rhodes, 2009) all belonging to Nontuberculous Mycobacteria (NTM) also designated as Mycobacteria other than Tuberculosis (MOTT), or Atypical Mycobacteria (ATM) (Primm et al., 2004; Al-Ghafli and Al-Hajoj, 2017). Among them, Mycobacterium fortuitum, $M$. marinum and $M$. chelonae are the most frequently isolated NTM from fish (Decostere et al., 2004).

Mycobacteriosis in ornamental fish is characterized by non specific clinical signs that include progressive emaciation, chronic non healing ulcers, pigmentary changes, scale loss and death of fish over a period of months to years. Often, the infection is clinically inapparent and may not produce clinical signs (Gauthier and Rhodes, 2009; Floyd, 2013). Granulomatous inflammation is the classic histopathological manifestation of piscine mycobacteriosis which is grossly visible as grey or white nodules in multiple internal organs (Chinabut, 2009), especially in the kidney and the spleen. Significant variation in the size and structural organization of granulomas are seen, from highly organized lesions with concentric layers of epithelioid cells to poorly organized inflammation (Gauthier and
Rhodes, 2009). Diagnosis is based on history, gross pathology, microscopic evaluation of organ smears for acid fast rods (AFR) followed by cultural, histopathological and molecular investigations.

This disease is especially a problem for ornamental fish industry due to several reasons. The presence of clinical disease directly affects their physical appearance, thriftiness and the reproductive performance resulting in economic losses to the ornamental fish industry. Further, the disease will slowly and silently spread among the fish in a farm due to the longevity of the ornamental fish and the chronic nature of the infection that transmits both horizontally and vertically as well as through water, making it difficult to get rid of the infection once established. Further, the presence of mycoabcteriosis can adversely affect the international trade of aquarium fish (Passantino et al., 2008). Moreover, many of the NTM associated with fish infections are reported to cause cutaneous nodular and ulcerative lesions in humans (Caputo et al., 2010) posing a public health risk to those who handle aquarium fish or inadvertently get exposed to aquarium water contaminated with mycobacteria.

In the recent past, there have been published reports on the occurrence of mycobacteriosis in Sri Lankan ornamental fish. Both slow growing and fast growing Mycobacteria have been isolated from the internal organs of apparently healthy and clinically diseased fish collected from farms located in different districts of Sri Lanka (Dissanayake et al., 2009; Dissanayake et al., 2017). Perera et al (2010) identified $M$. fortuitum as the commonest species isolated from guppies showing clinical disease based on PCR-RFLP of the $r p o B$ gene. Edirisinghe et al (2014) reported the occurrence of NTM in aquarium water collected from different locations and M. fortuitum was the most frequently isolated species. 

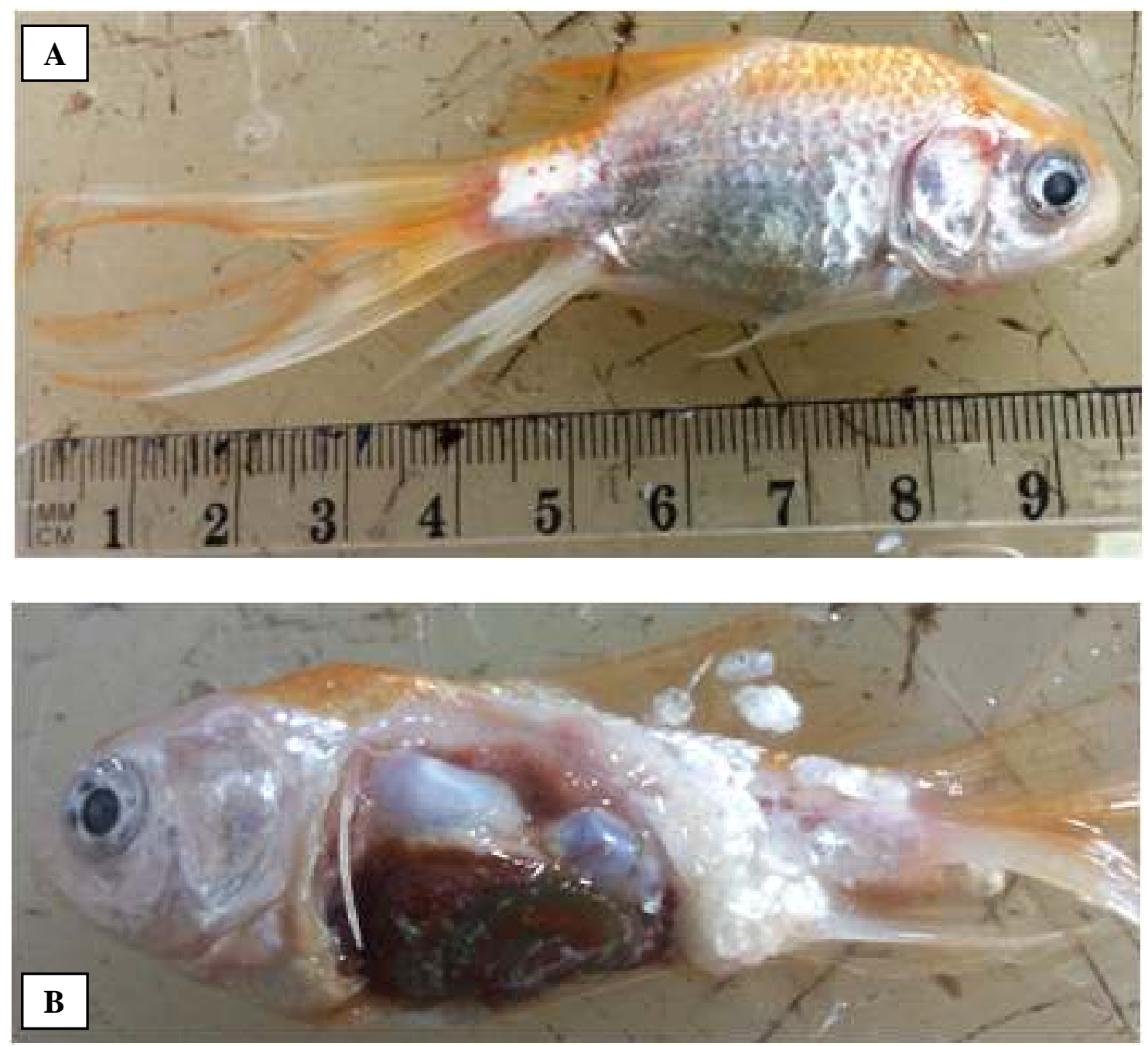

Figure 1: Case 1; A: A gold fish with abdominal distention, haemorrhages on the skin, scale loss and faded pigmentation. B: Viscera of the same gold fish showing numerous, white colour, variably sized granulomas

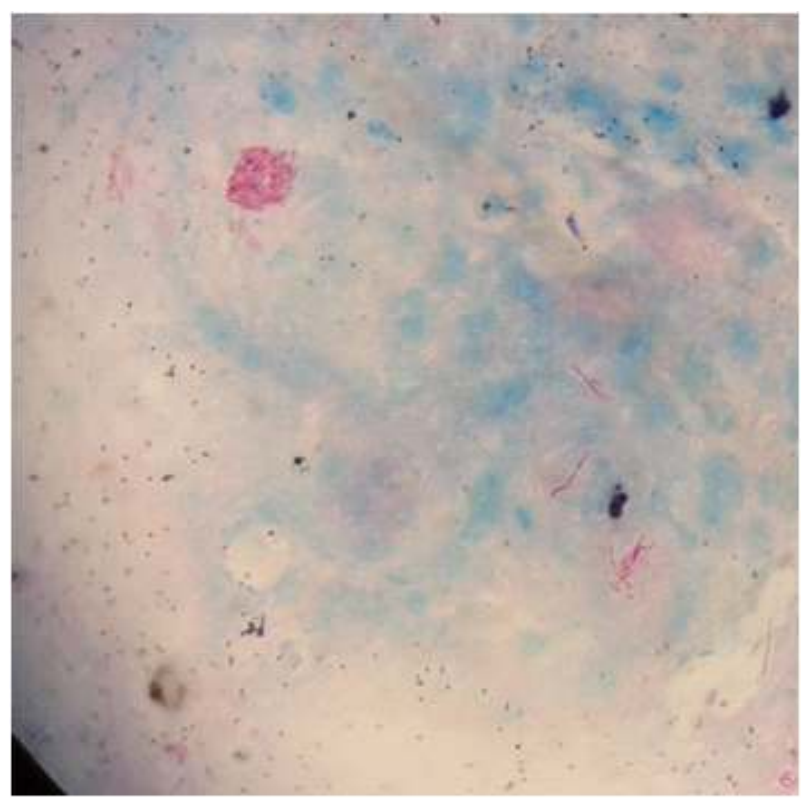

Figure 2 : Pink staining acid fast rods in a squashed tissue smear stained with ZN stain 
Piscine mycobacteriosis in gold fish and koi carp
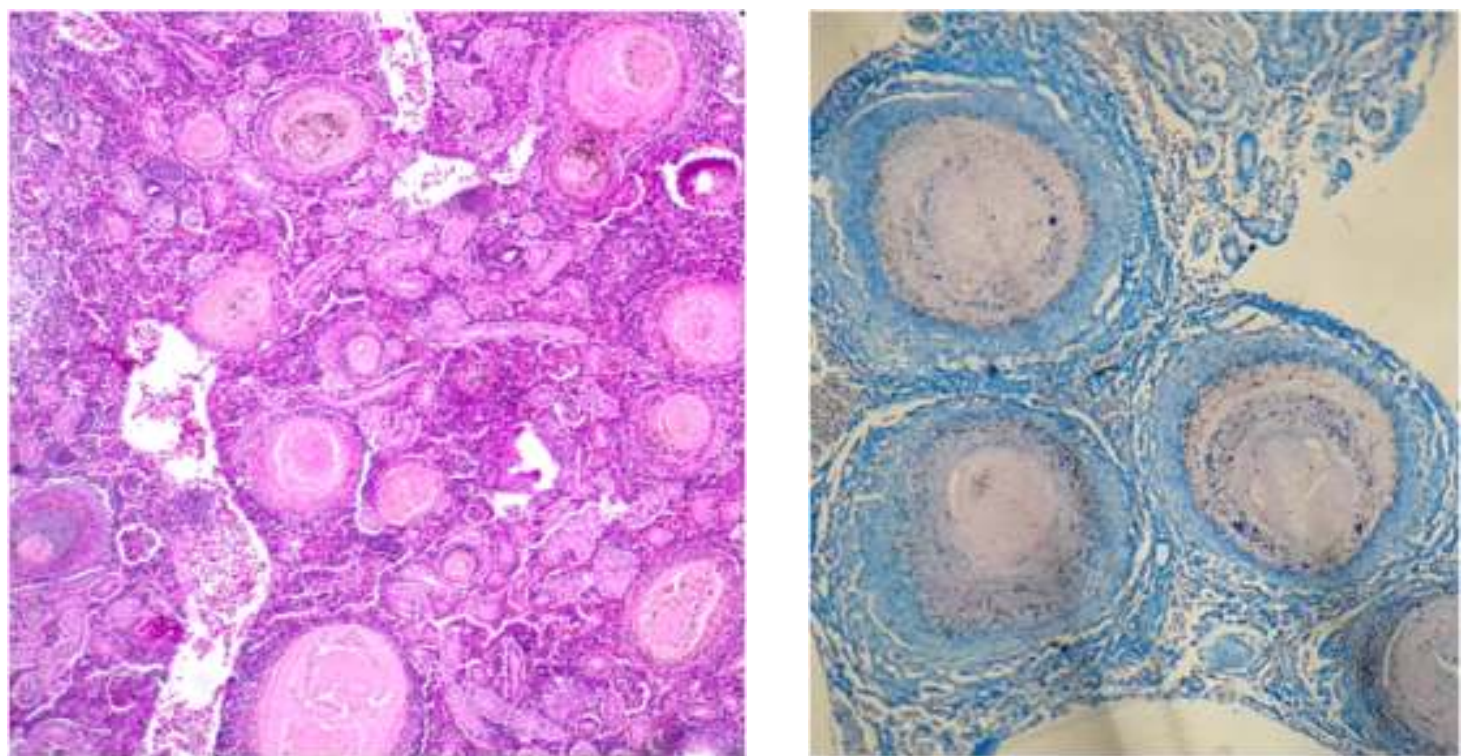

Figure 3: A: Histological section of the kidney of a gold fish from case 1 showing numerous granulomas with eosinophilic necrotic centers (H\&E stain) B: Histological section of the kidney of the koi carp from case 2 showing granulomas with central necrotic cores (ZN stain)

M

2

3

4

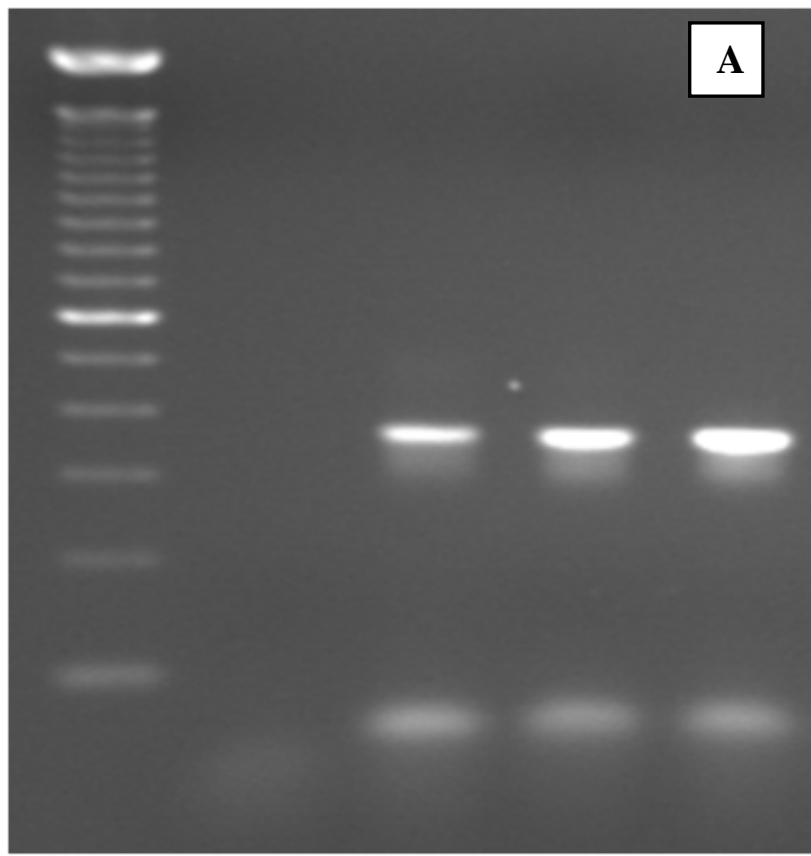

Figure 4 A: 360bp PCR amplicons of rpoB gene of mycobacteria; Lane M: DNA size marker (100bp ladder). Lane 1: Negative control, Lane 2: Positive control, Lane 3: PCR product of case No 1; Lane 4: PCR product of case No. 2
$\begin{array}{llll}M 1 & 1 & 2 & M 2\end{array}$

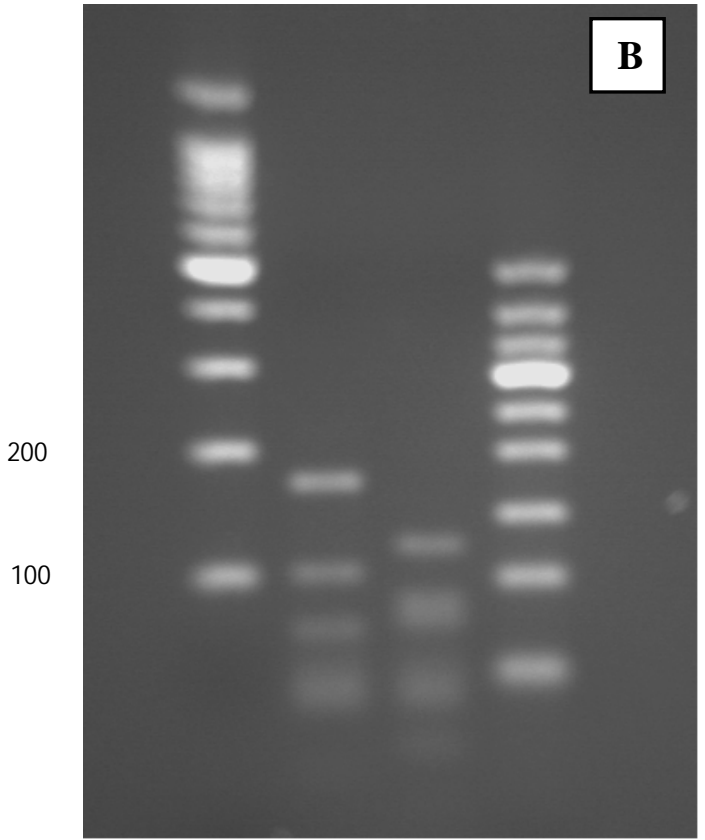

Figure 4 B: $M s p \mathrm{I}$ and HaeIII restriction enzyme digestion patterns of rpoB gene of $M$. fortuitum. Lane M1: DNA size marker (100bp ladder), Lane 2: MspI digestion pattern $(175,100,70)$ of $M$. fortuitum type I, Lane 2: Hae III digestion pattern $(120,90,80)$ of $M$. fortuitum type I; Lane M2: DNA size marker (50bp ladder) 
In this report, we describe the clinical manifestations, gross and histopathological lesions associated with mycobacteriosis in a group of gold fish that had been maintained in a fiberglass tank in a fish disease diagnosis laboratory, and a koi carp reared in a home garden pond.

\section{CASE PRESENTATION}

Case 1: A group of thirty gold fish (Carassius auratus) purchased from a fish breeding center had been maintained in a fiberglass tank in the Center for Aquatic Animal Disease Diagnosis and Research (CAADDR), to be used in an experiment once they attain maturity. They were fed with commercial pellet feed and the water changes were done weekly. After about one month, low grade mortality was observed in the group, and a maximum of 1-2 fish died per day that continued over a period of three weeks. The moribund fish displayed erratic swimming, lethargy, slight to moderate abdominal distention, scale loss, haemorrhages on the skin, faded pigmentation and reduced appetite (Figure 1A). Clinical signs were not consistent in all moribund fish and some died suddenly without showing any signs of the disease.

In March 2016, five moribund fish showing typical signs of the condition were collected from the affected tank and subjected to a detailed necropsy to find out the aetiological agent involved. The fish were euthanized using buffered MS-222 at a dose rate of $150 \mathrm{mg} / \mathrm{L}$. At necropsy, there were numerous, white, small, miliary granulomas in the kidney, liver, spleen, intestines and gonads (Figure 1B). These visceral granulomas were sharply demarcated from surrounding parenchymatous tissues. Mild ascites (accumulation of blood tinged fluid) was also observed in all five fish examined. For routine bacterial isolations, samples from kidney were cultured on trypticase soy agar and incubated overnight at room temperature. Since the gross pathology was suggestive of piscine mycobacteriosis, squashed tissue smears from the kidney, liver and spleen, and a smear of intestinal contents were prepared and stained with Ziehl Neelsen stain to look for acid fast rods (AFR).

Pieces of tissues from internal organs with granulomas were fixed in $10 \%$ buffered formalin and processed routinely for histological evaluation. Sections were stained using both hematoxylin and eosin (H\&E staining) and Ziehl Neelsen (ZN) stain. For the culture of mycobacteria, tissue samples of liver, kidney, intestine, gonads and heart were collected aseptically from each fish, transferred into sterile $1.5 \mathrm{ml}$ microcentrifuge tubes, homogenized and decontaminated by adding an equal volume of $1 \mathrm{M} \mathrm{NaOH}(\mathrm{w} / \mathrm{v})$ and incubated for 30 minutes at $37^{\circ} \mathrm{C}$ according to a procedure described previously (Dissanayake et al., 2009). Decontaminated samples were cultured on Ogawa egg medium and incubated aerobically at ambient temperature for two months. Inoculated media were observed daily for 7 days and then on every other day during rest of the incubation period.
For the confirmation of the species of Mycobacteria, total DNA was extracted from the isolate by QIAamp DNA mini kit (Qiagen, Germany) and a fragment of $360 \mathrm{bp}$ of the $r p o B$ gene was amplified by PCR according a protocol described by Lee et al. (2000). After verification of PCR amplicons by gel electrophoresis (1\% agarose gel stained with ethidium bromide), PCR product was subjected to Restriction Fragment Length Polymorphism (RFLP) analysis with the restriction enzymes Msp I and Hae III according to protocol described by Lee et al (2000). Further, PCR amplicons were sequenced and the resulting consensus sequences were aligned with public nucleotide sequences by nucleotide BLAST analysis.

Examination of the wet mounts of skin scrapings and gill clips revealed only few gill flukes (Dactylogyrus spp). Mesophilic, motile Aeromonas spp. was isolated from the kidney on TSA. AFR were observed in the kidney, liver, spleen of two fish examined. In others, none of the tissue smears other than smear of intestinal contents had AFR. In histopathological sections of kidney, liver and the spleen, multiple granulomas of varying sizes with eosinophilic necrotic centers were observed (Figure 3A), but AFR were either absent or rarely visible within those (Figure 3B). Off white colonies suggestive of mycobacteria appeared 7 days post inoculation in the kidney and liver samples. These colonies were $\mathrm{ZN}$ positive, hence sub cultured on Ogawa egg medium and identified by the morphology (growth rate and pigment production) and biochemical characteristics (Nitrate reduction, Urease, growth on MacConkey agar without Crystol Violet, Tween 80 hydrolysis, catalase and $\mathrm{NaCl}$ tolerance). $r p o B$ gene PCR confirmed the genus level identification as Mycobacterium yielding an amplicon of 360 bp (Figure 4A). Both phenotypic characteristics and PCR-RFLP patterns (Figure 4B) supported the identification of the isolate as $M$. fortuitum. The sequence comparisons revealed 99\% homology to Mycobacterium fortuitum subsp.fortuitum (ATCC 6841).

Case 2: A koi carp was presented to CAADDR with the complaints of lethargy, reduced appetite and erratic swimming. This koi carp was the only survivor out of fifteen koi carps (Cyprinus carpio koi) that had been maintained in a garden pond of a hobbyist. This garden pond was an established one with no recent introductions of new fish and chlorinated municipal water was used for water changes. According to the owners, low grade mortality (2-3 fish per day on some days) was observed that continued over a period of one week and the moribund fish showed erratic swimming, reduced appetite, lethargy and labored breathing with gasping air at the water surface. Owners tried to treat the fish with antibiotics and other chemicals, but all fish except the submitted koi carp succumbed to death. Upon submission to the laboratory, wet mounts of skin scrapings and gill clips examined revealed a heavy infestation of Trichodina spp., (12-15 per field) and Dactylogyrus spp., (gill flukes). Zeihl Neelson stained smear of fresh faeces showed 
Piscine mycobacteriosis in gold fish and koi carp

numerous acid fast rods. The fish was transferred to a well aerated treatment tank and maintained in a bath of $2 \%$ $\mathrm{NaCl}$ and $0.5 \mathrm{mg} / \mathrm{L}$ Potassium permanganate for a period of three days. On the third day of admission, fish was found dead. Necropsy and the subsequent investigations performed were markedly similar to case No. 1. At necropsy, a considerable amount of clear ascitic fluid in the abdominal cavity and very few atypical nodular lesions that were not well demarcated from the surrounding parenchymatous tissues were observed in the kidney, liver and spleen. Visceral organs were soft and irregular. Squashed smears made from the kidney, liver and the spleen showed clumps of AFB (Figure 2). Histopathological lesions included mild to moderate granulomatus inflammation, but the number of organized granulomas were markedly low compared to case No: 1. Mesophilic, motile Aeromonas spp., was isolated from the kidney of this fish as well. Off white colonies suggestive of mycobacteria appeared 6 days post inoculation in kidney and liver samples. This isolate was subsequently identified phenotypically, biochemically and later by PCR-RFLP as M. fortuitum. The amplified sequence matched Mycobacterium fortuitum subsp. fortuitum with $99 \%$ sequence identity.

\section{DISCUSSION AND CLINICAL SIGNIFICANCE}

M. fortuitum was first isolated from the tropical fish neon tetra in 1953 (Ross and Brancato, 1959). It has since been reported in different species of ornamental fish including Cyprinids and has received a remarkable attention as a fish pathogen. Gold fish and koi carps are two commonly cultured ornamental fish varieties in Sri Lanka and therefore Mycobacterium infections in such species could result in devastating impacts on the local sales as well as exports of ornamental fish. Sri Lanka imports ornamental fish brood stocks to improve the quality of existing stocks, from countries where piscine mycobacteriosis is prevalent, increasing the risk of transboundary transmission of this disease. However, it is not yet clear whether this disease has been introduced to Sri Lanka unintentionally through fish imports or it was there unnoticed and undiagnosed. Therefore, considering the economical importance of the ornamental fish industry and associated international trade, early and accurate diagnosis of mycobacterial infections and subsequent management of affected stocks should be considered as crucial aspects in ornamental fish health management.

The two cases described in the present study show two different clinical manifestations which were non pathognomonic of the infection posing a diagnostic challenge. Routine laboratory diagnosis of piscine mycobacteriosis at the field largely depends on the examination of visceral organ smears with bench top $\mathrm{ZN}$ staining for AFR. However, as observed in both these cases, AFR may not be visible in all affected fish even in the presence of granulomatous lesions in visceral organs. It has been reported that mycobacteria can be detected in infected tissue either in the very early or in the later stages of infection. Another diagnostic dilemma associated with mycobacterisos is the lack of concordance between the presence of AFR in organ smears and positive culture isolation results. In both these cases, irrespective of the presence of AFR, it was possible to isolate M. fortuitum from the kidney and liver tissues. Negative growth results in some organ samples may be either due to a low number of live mycobacteria which may have been killed during decontamination (Shukla et al., 2013). However, on the other hand, in the absence of clinical signs and granuloma, isolation of mycobacteria from fish tissues may also be a result of mere colonization of internal organs with mycobacteria present in aquatic environment.

Unfortunately, there is no effective treatment for mycobacteriosis in fish. Depopulation of the affected stock followed by disinfection of tanks is the only reliable means of control. Treatment with antimycobacterial drugs is not advocated since the full disease resolution takes time and promotes the resistance development. Antibiotic treatment would not be cost effective for the farmers unless for individual, valuable fish (Strike et al., 2017). The remaining fish of case no: 1 are being maintained in isolation and antibiotic treatment was not attempted. In case no: 2 , the owners were advised to disinfect and dry the pond.

Maintaining a population of Mycobacterium positive ornamental fish stock makes a little sense to ornamental fish farmers since they are debilitated and prone to have chronic health problems and poor performance in terms of growth and reproduction. Infected fish will be a constant source of Mycobacteria to other fish inhabiting the same tank and also to their offsprings (Floyd, 2013). These two cases in general support two important phenomena. First, ornamental fish infected with mycobacteria die off mostly not due to the pimary pathogen but due to secondary infections by other bacteria and parasites and poor water quality conditions that make them stressful. And secondly, any fish from a population experiencing low grade mortality accompanied by emaciation, faded pigmentation and weight loss should be suspected for mycobacteriosis. In intensive culture of ornamental fish, high organic loads, poor water quality and high stocking density exacerbate the condition. Adoption of good husbandry practices will minimize the risk of infection.

Piscine mycobacterisis is a minor zoonosis. Given the fact that ornamental fish and aquarium water are natural reservoirs of pathogenic NTM, the close contact between aquarium fish and people offers favourable conditions for mycobacterial transmission (Kušar et al., 2017). Therefore, it is of prime importance to educate those involved in ornamental fish farming; farmers, aquaculturists, hobbyists and veterinarians on the potential zoonotic risk associated with exposure to ornamental fish infected with mycobacteria. 


\section{ACKNOWLEDGEMENT}

Financial support given by National Science Foundation (RG/2016/BT/03) is acknowledged. Authors would like to thank Mr. Sampath Bandara and Mrs. Waruni Jayaweera for their support during the laboratory analyses.

\section{REFERENCES}

Antonio, D. B., Swanson, C., Cech, J. J., Mager, R. C., Doroshov, S., Hedrick, R. P. (2000). Prevalence of Mycobacterium in wild and captive delta smelt. California Fish and Game, 86: 233243.

Caputo, V., Fiorella, S. and Orlando, E. (2010). Sporotrichoid cases of Mycobacterium marinum skin Infection. Clinical Medicine Insights: Dermatology, 3: 2529.

Chinabut, S., Limsuwan, C. and Chanratchakool, P. (1990). Mycobacteriosis in the snakehead, Channa striatus (Fowler). Journal of Fish Diseases, 13: 531535. doi:10.1111/j.1365-2761.1990.tb00813.x

Decostere, A., Hermans, K. and Haesebrouck, F. (2004). Piscine Mycobacteriosis: A Literature Review Covering the Agent and the Disease It Causes in Fish and Humans. Veterinary Microbiology, 99: 3-4: 159-66. DOI: 10.1016/j.vetmic.2003.07.011.

Dissanayake, D. R. A., Perera, V., Jagoda, S. S. S. de.S., Edirisinghe, E. A. R., Arulkanthan, A. (2017). Characterization of Non-tuberculous Mycobacteria Isolated from Apparently Healthy and Diseased Fresh Water Ornamental Fish in Sri Lanka. Asian Fisheries Science, 30:118129.

Dissanayake, D. R. A., Wijewardana, T. G., Arulkanthan, A., Buddhika, M. D. P. and Jagoda, S. S. S. de.S. (2009). Occurrence of mycobacteria in various organs of clinically affected and apparently healthy guppies (poecilia reticulate). Proceedings of the Peradeniya University Research sessions.

Edirisinghe, E. A. R., Dissanayake, D. R. A., Abayasekera, C. L., Arulkanthan, A. (2014). Occurrence of nontuberculous mycobacteria in aquatic sources of Sri Lanka. International Journal of Mycobacteriology, 3 (4); 242-246. doi: 10.1016/j.ijmyco.2014.10.007.

Floyd, R. F. (2013). Mycobacterial Infections of Fish. SRAC Publication. 4706.

Gauthier, D.T., Rhodes, M. W. (2009). Mycobacteriosis in fishes: a review. The Veterinary Journal, 180: 33-47. doi: 10.1016/j.tvj1.2008.05.012.

Al-Ghafli, H. and Al-Hajoj, S. (2017). Nontuberculous Mycobacteria in Saudi Arabia and Gulf Countries: A Review. Canadian Respiratory Journal, Article ID 5035932. doi:10.1155/2017/5035932

Jacobs, J. M., Stine, C. B., Baya, A. M., Kent, M. L. (2009). A review of mycobacteriosis in marine fish. Journal of Fish Diseases, 32:119-130. doi: 10.1111/j.1365-2761.2008.01016.x.

Kušar, D., Zajc, U., Jenčič, V., Ocepek, M., Higgins, J., Žolnir-Dovč, M. and Pate, M. (2017). Mycobacteria in aquarium fish: results of a 3-year survey indicate caution required in handling pet-shop fish. Journal of Fish Diseases, 40: 773784. doi:10.1111/jfd.12558

Lee, H., Park, H. J., Cho, S. N., Bai, G. H. and Kim, S. J. (2000). Species identification of mycobacteria by PCRrestriction fragment length polymorphism of the $r p o B$ gene. Journal of Clinical Microbiology, 38: 2966-2971.

Passantino, A., Macrı, D., Coluccio, P., Foti, F., Marino F. (2008). Importation of mycobacteriosis with ornamental fish: Medico-legal implications. Travel Medicine and Infectious Disease, 6:240244. doi: 10.1016/j.tmaid.2007.12.003.

Perera, P. D. V. M., Dissanayake, D. R. A., Arulkanthan, A., Jagoda, S. S. S. de S., Edirisinghe, E. A. R. (2010). Identification of fish pathogenic Mycobacterium species by PCR restriction fragment length polymorphism of the $r p o B$ gene. Proceedings of the Peradeniya University Research Sessions. 15: 320322.

Primm, T. P., Lucero, C. A., Falkinham, J. O. (2004). Health impacts of environmental mycobacteria. Clinical Microbiology Reviews, 17: 98-106. doi:10.1128/CMR.17.1.98-106.2004.

Ross, A. J., Brancato, F. P. (1959). Mycobacterium fortuitum cruz from the tropical fish Hyphessobrycon innesi. Journal of Bacteriology, 78(3): 392-395.

Shukla, S., Sharma, R. and Shukla, S.K. (2013). Folia Microbiologica, 58: 429. doi:10.1007/s12223-0130225-y

Strike, T. B., Feltrer, Y., Flach, E., Macgregor, S. K. and Guillaume, S. (2017). Investigation and management of an outbreak of multispecies mycobacteriosis in Australian lungfish (Neoceratodus fosteri) including the use of triple antibiotic treatment. Journal of Fish Diseases, 40: 557570. Doi:10.1111/jfd.12535 